\title{
INVESTIGAÇÃO QUALITATIVA: PERSPETIVA GERAL E IMPORTÂNCIA PARA AS CIÊNCIAS DA NUTRIÇÃO
}

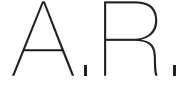
ARTIGO DE REVISÃO

1 Unidade Local de Saúde de Matosinhos, Serviço de Nutrição e Alimentação, Rua Dr. Eduardo Torres, 4464-513 Sra. Hora, Matosinhos, Portugal

2 Faculdade de Enfermagem da Universidade Estadual de Campinas,

Rua Tessália Vieira de Camargo, n. ${ }^{\circ} 126$ Cidade Universitária "Zeferino Vaz", Campinas, SP, Brasil

${ }^{3}$ Pontificia Universidade Católica de Minas Gerais, Campus Poços de Caldas, Av. Padre Francis Cletus Cox, n. ${ }^{\circ} 1661$, Country Club, Poços de Caldas, MG, Brasil

*Endereço para correspondência: Isabel Ferraz Pinto Unidade Local de Saúde Serviço de Nutrição e Alimentação,

Alimentaça,

Rua Dr. Eduardo Torres,

(4045)

monday.isabel@gmail.com

Histórico do artigo:

Recebido a 5 de março de 2018 Aceite a 25 de setembro de 2018

\section{QUALITATIVE RESEARCH: GENERAL PERSPECTIVE AND IMPORTANCE FOR THE NUTRITION SCIENCES}

Isabel Ferraz Pinto"; Claudinei José Gomes Campos²; Cibele Siqueira ${ }^{3}$

\section{RESUMO}

Este artigo apresenta, de forma simples, a definição e fundamentos da pesquisa qualitativa, de forma a introduzir a temática da importância desta metodologia de investigação, para as ciências da nutrição e alimentação e os nutricionistas. Ao contrário da metodologia quantitativa, a pesquisa qualitativa é realizada através de uma abordagem interpretativa e naturalística do tema de estudo. Com o foco nos significados atribuídos pelos indivíduos ao objeto de estudo, a investigação qualitativa oferece um percurso científico propício à compreensão de um fenómeno tão complexo como o alimentar e nutricional. Não obstante o atual reconhecimento científico da importância da evidência qualitativa, a utilização desta metodologia é ainda muito reduzida e/ou pouco valorizada. 0 estabelecimento de mecanismos de educação e formação nesta temática e a elaboração de processos de colaboração interdisciplinar na realização de estudos científicos tornam-se assim vitais para o desenvolvimento da utilização desta metodologia, na área das ciências da nutrição e alimentação.

\section{PALAVRAS-CHAVE}

Metodologia, Nutrição e Alimentação, Pesquisa qualitativa

\section{ABSTRACT}

This article presents, in a simple way, the definition and foundations of the qualitative research in order to introduce the thematic of the importance of this research methodology for the nutritional sciences and nutritionists. Unlike the quantitative methodology, qualitative research is realized through an interpretative and naturalistic approach of the subject of study. Qualitative research offers a scientific pathway conducive to the understanding of a phenomenon as complex as food and nutrition, however the use of this methodology is still very reduced or little valued. The establishment of education / training mechanisms in this area and processes of interdisciplinary collaboration in the conduct of scientific studies thus becomes vital for the development of the use of this methodology in the area of nutrition and food sciences.

KEYWORDS

Methodology, Food and Nutrition, Qualitative research

\section{INTRODUÇÃO}

A pesquisa qualitativa originária das ciências humanas e sociais, ganhou, nas últimas décadas, no seio das ciências da saúde, força e valor, através do reconhecimento da sua contribuição para uma mais profunda compreensão da complexidade do comportamento humano e da interação entre doença e sociedade, levando alguns autores a anunciar "uma revolução qualitativa", na investigação científica em saúde (1-3). Na verdade, esta abordagem metodológica, anteriormente posicionada nos lugares inferiores da hierarquia de produção de evidência científica, é, atualmente, cada vez mais considerada como parte importante dos processos de tomada de decisão, que direcionam o desenvolvimento de políticas e práticas de saúde (2).

Os resultados produzidos por este tipo de metodologia não envolvem procedimentos estatísticos. A pesquisa qualitativa tende a colocar questões utilizando "o quê", "como" e "porquê", e a analisar as respostas no contexto da vida quotidiana e dos significados e explicações atribuídos por cada indivíduo ao fenómeno estudado (4). Das inúmeras áreas de valor da pesquisa qualitativa para a saúde salientam-se a sua relevância para a compreensão das experiências e sentimentos dos pacientes e seus familiares e para o estudo da organização dos serviços de saúde (3). De destacar, também, a sua importância para a análise crítica do exercício e formação dos profissionais de saúde, e o seu valor para o melhor planeamento, condução e execução de desenhos de estudo quantitativo (3). No entanto, este tipo de pesquisa reúne ainda muitas resistências e dúvidas quanto aos seus tipos, características e aplicações. Muitos consideram também difícil aceder à terminologia que a mesma envolve. E não obstante o atual 
reconhecimento científico da importância da evidência qualitativa, a abordagem quantitativa continua a dominar os estudos desenvolvidos, o que se publica e lê e, por conseguinte, o que se ensina e discute ao nível académico $(4,5)$.

No caso específico das ciências da nutrição e alimentação, o panorama não é diferente. Para muitos nutricionistas, a palavra investigação contempla maioritariamente perguntas, procedimentos e desenhos de estudo inerentes à pesquisa quantitativa (2-4). Contudo, quando um fenómeno não é facilmente medível, ou os processos de uma intervenção precisam ser compreendidos em profundidade, ou o conhecimento sobre um grupo ou cultura é limitado, ou as razões sobre determinados resultados quantitativos necessitam ser discernidas, outro tipo de investigação é necessário. Nestes casos, a melhor abordagem é a oferecida pela pesquisa qualitativa (1-4).

Com ênfase no significado atribuído pelos indivíduos e na compreensão profunda dos fenómenos estudados, a pesquisa qualitativa pode, assim, complementar o conhecimento oferecido pela pesquisa quantitativa, ao explicar, por exemplo, padrões de comportamento ou acontecimentos que parecem "irracionais", inexplicáveis ou opacos, sob uma perspetiva biomédica (4). São exemplos, os estudos qualitativos realizados para revelar os aspetos subjetivos do processo de tratamento ambulatorial da obesidade $(6,7)$, a contribuição deste tipo de pesquisa para o conhecimento dos determinantes psicológicos, sociais, culturais do comportamento alimentar (8), o uso da abordagem qualitativa para uma melhor caracterização da experiência alimentar de pacientes em ambiente hospitalar (9), entre tantas outras áreas de valor já identificadas (3).

No entanto, ao mesmo tempo em que a pesquisa qualitativa parece oferecer um percurso científico propício à compreensão de um fenómeno tão complexo como o alimentar e nutricional, a utilização desta metodologia é ainda muito reduzida ou pouco valorizada, no âmbito das ciências da nutrição (1-4). Assim, é objetivo deste artigo apresentar, de forma simples, a definição e fundamentos da pesquisa qualitativa, de modo a introduzir a temática da importância da investigação qualitativa, para as ciências da nutrição e alimentação e para os nutricionistas.

\section{A PESQUISA QUALITATIVA \\ Definição e Características}

A investigação qualitativa tem um foco multimetodológico, envolvendo abordagens interpretativas e naturalísticas dos temas estudados. $\bigcirc$ pesquisador qualitativo investiga num ambiente natural e interpreta os fenómenos, segundo os significados atribuídos pelos participantes (10). Esta definição ressalta pontos importantes relativos a este tipo de metodologia, destacando-se entre eles, a abordagem multimetodológica, que envolve a utilização de narrativas, estórias de vida, documentos históricos, entrevistas, observações, fotografias, textos visuais, entre outras fontes de dados. Do mesmo modo, chama também a atenção para outras particularidades, como a utilização do contexto natural, entendido como o local onde as pessoas vivem ou desenvolvem suas atividades quotidianas, em contraposição aos estudos que controlam este ambiente, e a valorização do significado e sentido que os participantes atribuem às coisas, entendidas aqui como o objeto de estudo (10).

Minayo define a metodologia qualitativa como:

"aquela capaz de incorporar a questão do significado e da intencionalidade como inerentes aos atos, às relações, e às estruturas sociais, sendo estas últimas encaradas tanto no seu advento quanto na sua transformação, como construções humanas significativas" (11).

Dentro desta conceção, voltada para estrutura social do fenómeno, a autora ressalta ainda que, "a pesquisa qualitativa preocupa-se com o universo de significados, motivos, aspirações, crenças, valores e atitudes, o que corresponde ao universo mais profundo das relações, dos processos e dos fenómenos, que não podem ser reduzidos à operacionalização de variáveis" (11).

Por fim, destaca-se a palavra significado, explícita nas duas definições anteriores, como ponto-chave dentro da abordagem qualitativa, pois através da sua interpretação podem-se compreender o participante da pesquisa, o seu modo de vida e as suas relações interpessoais (10).

A investigação qualitativa tem, na sua essência, quatro características, que se encontram resumidas em seguida.

1. A fonte direta dos dados é o ambiente natural e o investigador é o principal agente da sua colheita

$\mathrm{O}$ ambiente e o contexto onde os indivíduos vivem quotidianamente e criam as suas ações e modus vivendi, têm importância essencial na compreensão significativa das suas atividades. O meio imprime ao indivíduo que nele vive, traços peculiares que são desvelados à luz da compreensão dos significados construídos por ele próprio (10). Desta maneira, compreender o indivíduo fora do seu contexto natural, pode criar situações artificiais produzindo interpretações equivocadas. Como os problemas são pesquisados no ambiente natural em que ocorrem, sem qualquer manipulação intencional do pesquisador, chamamos estes estudos de "naturalísticos" (11).

O pesquisador, presente no ambiente do estudo, torna-se de alta importância para a pesquisa, visto que o mesmo sofre a ação direta desse ambiente, e o fenómeno estudado passa a ser interpretado e compreendido de maneira mais abrangente (12). O pesquisador qualitativo deve manter-se no ambiente de estudo por tempo prolongado, de modo a proporcionar espaços deliberados para o aparecimento de conteúdos e perceber aspetos imprevisíveis de antemão (13). No ambiente natural, dentro de um enquadramento qualitativo de pesquisa, o pesquisador serve, assim, como 'instrumento' através do qual os dados são coletados (14).

2. Os dados recolhidos são essencialmente de caráter descritivo

Os dados são coletados e apresentados na sua quase totalidade na forma de texto ou imagens. Estes dados incluem transcrições de entrevistas, anotações de campo, fotografias, produções pessoais, depoimentos ou outras formas de documentos. O investigador qualitativo tenta interpretar os dados em toda a sua riqueza, respeitando, se possível, a forma de registo ou transcrição. Citações ou trechos dos documentos coletados são normalmente apresentados para ilustrar uma situação ou esclarecer uma afirmação (15).

3. O investigador interessa-se, acima de tudo, por tentar compreender o significado que os participantes atribuem às suas experiências

A atenção do pesquisador está depositada na ênfase que os indivíduos colocam no seu modo de vida, nas suas relações quotidianas, e em como significam e ressignificam as mudanças ou manutenção de determinados costumes ou crenças. O significado ou sentido que os indivíduos dão aos fenómenos vivenciados é o foco da pesquisa qualitativa. A maneira como os participantes vivenciam e informam uma situação vivida é relevante e singular. Deste modo, o enfoque dos dados pesquisados reside nos significados atribuídos pelos participantes (15). 4. A análise dos dados é feita de forma indutiva

A indução é um método mental por intermédio do qual, partindo-se de dados particulares, suficientemente constatados, se infere uma conclusão geral ou universal, não contida nas partes examinadas. Desta forma, o objetivo dos argumentos indutivos é apresentar resultados, cujo conteúdo é mais amplo do que as premissas nas quais se basearam (16). Não há aqui a preocupação de comprovar hipóteses. Através da inter-relação dos dados à medida que são colhidos, os pesquisadores 
qualitativos vão construindo, de "baixo para cima", as suas conclusões e teorias. A construção do resultado final do estudo vai assim ganhando forma à medida que os dados vão sendo analisados, não se conhecendo de antemão qual é a sua forma ou conteúdo (12).

Cabe referir também que, no âmbito da investigação qualitativa, vários métodos de pesquisa podem ser utilizados. São exemplos: a etnografia, a fenomenologia, a teoria baseada em dados, o estudo de caso qualitativo, a interação simbólica e a pesquisa ação (15). O método escolhido para determinada investigação reflete a abordagem geral às questões de pesquisa em estudo, e no âmbito de cada método uma variedade de técnicas de amostragem e colheita de dados podem ser usados. Os estudos qualitativos utilizam, amostras pequenas e não padronizadas, quando comparados com os estudos quantitativos. A composição da amostra é, assim, muitas das vezes realizada por intencionalidade, o que significa que os pesquisadores qualitativos procuram propositadamente aqueles indivíduos que, segundo observação ou vivência prévia do campo de estudo, possam fornecer dados importantes e ricos sobre o fenómeno, contribuindo assim para que se possam atingir os objetivos de pesquisa propostos (17).

\section{As Técnicas de Colheita de Dados Adequadas aos Estudos Qualitativos}

As principais técnicas utilizadas na colheita de dados qualitativos são a entrevista não estruturada, a observação e o grupo focal (18). O pesquisador deve atentar a todos os elementos recolhidos, pois um aspeto aparentemente trivial pode ser importante para a melhor compreensão do problema. As técnicas de colheita de dados devem ter validação científica e revelar as condições metodológicas utilizadas, sendo esta uma necessidade ainda mais premente na metodologia qualitativa, muitas vezes ainda vista, equivocadamente, como menos científica ou rigorosa (19).

\section{A Entrevista}

A entrevista é uma das técnicas mais utilizadas na colheita de dados, em pesquisa qualitativa. Trata-se a entrevista como um encontro entre duas pessoas, a fim de que uma delas obtenha informações a respeito de determinado assunto, mediante uma conversação de natureza profissional (20). Algumas vantagens desta técnica são a captação imediata e corrente de informações desejadas, praticamente com qualquer tipo de informante e sobre os mais variados assuntos, além da oportunidade de aprofundar tópicos levantados por outras técnicas de colheita, de alcance mais superficial (16). No que toca à diretividade da condução, as entrevistas mais adequadas ao caráter qualitativo de investigação, são as não estruturadas e as semiestruturadas. A primeira, de caráter totalmente aberto, coloca o entrevistado em posição de discorrer sobre um determinado assunto de forma livre, e é indicada quando o pesquisador tem pouca informação sobre o assunto ou sobre o participante da pesquisa. Já nas entrevistas semiestruturadas, o caráter de abertura dada às questões continua, porém há delimitação específica na temática, ou seja, são colocados elementos específicos na construção do enunciado da pergunta, que darão um certo direcionamento ao que se deseja conhecer com maior profundidade. $\mathrm{O}$ encadeamento das perguntas das entrevistas de caráter semiestruturado é apoiado pelos pressupostos e teorias que interessam à pesquisa, e simultaneamente fruto de novos pressupostos que surgem ao longo da entrevista (15).

\section{Observação Participante}

Nascida na tradição antropológica de investigação, a observação participante, inicialmente utilizada e batizada por Brosnilaw Malinovisk, também é amplamente utilizada em pesquisas qualitativas (21). Tal técnica permite o contacto direto entre pesquisador e o fenómeno observado, para se conseguir informações sobre a realidade dos participantes em seus contextos próprios. Esta técnica guarda a importância de se poder observar uma variedade de situações ou fenómenos não possíveis de revelar pelas entrevistas, possibilitando assim o acesso à imponderabilidade da vida real (22).

\section{Grupo Focal}

O grupo focal é uma técnica de colheita de dados qualitativos eficaz para esclarecer as normas sociais de uma comunidade ou subgrupo, bem como a gama de perspetivas que existem dentro dessa comunidade ou subgrupo, sobre determinado tema ou assunto (23). Os grupos focais procuram iluminar a opinião do grupo, permitindo o acesso a múltiplos pontos de vista sobre um tema específico. Uma das suas principais vantagens é a capacidade de produzir uma grande quantidade de informações num período relativamente curto de tempo, valorizando a dinâmica do grupo. A sensibilização dos participantes, a organização e composição do grupo, bem como a condução dos grupos focais são pontos relevantes para a correta utilização desta técnica (23).

\section{A Análise de Dados na Pesquisa Qualitativa}

Uma análise qualitativa fidedigna não prescinde dos verbos compreender e interpretar e dos substantivos experiência e vivência (20). Os métodos de análise em pesquisa qualitativa são vários, porém o pesquisador deve dominar a técnica, percorrer as fases com transparência, e apresentar cada passo de modo a conferir credibilidade à pesquisa. A experiência do pesquisador, o embasamento teórico e o domínio da técnica de análise de dados utilizada, interferem de modo importante, tanto nos resultados quanto nas conclusões. Em pesquisas qualitativas, o método mais utilizado para o tratamento dos dados é a análise de conteúdo.

As técnicas de análise de conteúdo permitem tornar replicáveis e válidas inferências sobre dados de um determinado contexto, por meio de procedimentos especializados e científicos (11). Existem várias modalidades de análise de conteúdo. Optamos aqui em descrever brevemente três tipos (11). Na análise temática como o próprio nome indica, o tema está ligado a determinado assunto, e pode ser representado por uma palavra, frase ou um resumo. A análise de discurso tem como foco principal o sentido de uma palavra, expressão ou uma proposição que não existe em si mesmo. Por fim, a análise lexical tem início com a contagem de palavras e após avança em direção da identificação e dimensão do texto em estudo. As palavras são agrupadas por similaridade, retirando-se as que apresentam pouco interesse, de modo que no final representem o sentido do texto (11). Atualmente, existem também diversas ferramentas informáticas de suporte à análise de dados realizada pelo pesquisador (11).

\section{Os Procedimentos de Rigor Metodológico na Pesquisa Qualitativa}

Apesar das diferenças dos objetivos e métodos utilizados na investigação quantitativa e qualitativa, a necessidade de conduzir e reportar estudos metodologicamente rigorosos e transparentes é comum a ambas as abordagens. Na pesquisa qualitativa, rigor é frequentemente avaliado utilizando o conceito de Confiabilidade (24). Miles e Huberman, com base no trabalho de Lincon e Guba, propuseram cinco elementos que devem ser aplicados para garantir a Confiabilidade: Credibilidade, Transferibilidade, Dependabilidade, Confirmabilidade e Autenticidade (25). As definições de cada um destes elementos encontram-se reunidas no Quadro 1. 
Quadro 1

Definição dos elementos de rigor na pesquisa qualitativa $(24,25,26)$

\begin{tabular}{ll}
\hline Credibilidade & $\begin{array}{l}\text { Reflete o valor de verdade do processo de colheita e } \\
\text { análise de dados, garantindo assim que as construções } \\
\text { do investigador reproduzem os fenómenos em estudo e os } \\
\text { pontos de vista dos participantes. }\end{array}$ \\
\hline Transferibilidade & $\begin{array}{l}\text { Reflete o grau em que os resultados do estudo podem ser } \\
\text { transferidos para outras situações. }\end{array}$ \\
\hline Dependabilidade & $\begin{array}{l}\text { Aborda a consistência dos dados, bem como a } \\
\text { possibilidade do estudo ser auditado. }\end{array}$ \\
\hline Confirmabilidade & $\begin{array}{l}\text { Garante que a colheita e análise dos dados foram } \\
\text { realizadas por um processo fiel, refletindo o fenómeno em } \\
\text { estudo e não as ideias ou desvios do investigador. }\end{array}$ \\
\hline Autenticidade & $\begin{array}{l}\text { Demonstra que o estudo conseguiu representar diferentes } \\
\text { realidades. }\end{array}$ \\
\hline
\end{tabular}

Uma série de estratégias tem sido sugerida para alcançar estes elementos, tais como: a reflexão do pesquisador sobre as suas crenças e percurso profissional prévio, a presença por tempo prolongado no ambiente de estudo, a verificação do processo de análise por um auditor externo, entre outras (26).

Do mesmo modo, o uso das recomendações atualmente preconizadas para a comunicação formal de estudos científicos é também bastante aconselhável aquando da escrita e publicação de pesquisas qualitativas. A utilização destas ferramentas favorece a melhoraria da qualidade da apresentação dos estudos e permite ao leitor um melhor entendimento da conduta do investigador quanto ao desenho do estudo, o processo de análise dos dados e de apresentação dos resultados (27). Atualmente está disponível na literatura um conjunto de recomendações, desenvolvidas para um grupo diverso de desenhos de estudo e áreas clínicas de intervenção quer quantitativas quer qualitativas (27). São exemplos para os estudos qualitativos, os COREQ - Consolidated criteria for reporting qualitative research e os SRQR - Standards for reporting qualitative research: a synthesis of recommendations $(28,29)$.

A Importância da Pesquisa Qualitativa para as Ciências da Nutrição Talvez a forma mais simplista de abordar o estudo da nutrição é entendê-la apenas como um processo biológico. No entanto, o fenómeno alimentar e nutricional é uma entidade amplamente mais complexa, do que a oferecida por esta abordagem. Ele reveste-se de características, papéis e nuances múltiplas, para o indivíduo e a sociedade, tais como as psicossociais, emocionais, culturais, político-económicas e organizacionais, entre muitas outras (1-4). Os nutricionistas trabalham diariamente no centro desta complexidade, tentando diminuir o intervalo entre os dados científicos lógicos e ambiguidade do comportamento alimentar humano, pressentindo o impacto maior e mais abrangente que alimentação tem na vida de um indivíduo, grupo ou sociedade ou equacionando o seu papel e prática profissional, no seu ambiente de trabalho.

A pesquisa qualitativa, quando realizada corretamente, oferece um caminho metodológico para o discernimento destas e muitas outras questões. Os estudos desenvolvidos nesta área começaram há algumas décadas, com as pesquisas de foro antropológico (30). Os antropologistas estudaram o simbolismo alimentar, os marcadores alimentares de identidade regional e nacional, as proibições e tabus, os rituais e organização social em torno da alimentação, entre muitas outras temáticas $(30,31)$.

Atualmente, na área da alimentação e nutrição, os tipos de objetos de estudo ampliaram-se. A pesquisa qualitativa tem-se tornado relevante para a compreensão, em profundidade, dos padrões de comportamento, incluindo os padrões de consumo alimentar, e como esses padrões podem influenciar e interagir com a saúde e o estado nutricional. Nesta base, este tipo de pesquisa é útil também na identificação de prioridades e necessidades relevantes para contextos sociais e culturais específicos e/ou grupos de indivíduos, e conceber e implementar intervenções nutricionais apropriadas (1-4).

Do mesmo modo, na literatura, são encontradas também pesquisas sobre: o estudo das dimensões sociais, cognitivas e psicológicas do comportamento alimentar, a avaliação de intervenções educativas, a análise da prática profissional do nutricionista, a avaliação da implementação de políticas e programas de alimentação e nutrição e de programas de educação graduada e pós-graduada, e a organização de serviços de alimentação coletiva e restauração $(30,31)$, entre muitas outras. A pesquisa qualitativa tem sido também utilizada para melhor entender os resultados produzidos pelas pesquisas de foro quantitativo, ou mesmo para colaborar no desenho de estudos quantitativos, abrindo espaço para o que hoje se denomina de pesquisa com metodologia mista ou "mixed methods studies"(32).

\section{ANÁLISE CRÍTICA E CONCLUSÕES}

Este artigo apresentou a definição e fundamentos da pesquisa qualitativa, de forma a introduzir a temática da importância desta metodologia de investigação, para as ciências da nutrição e alimentação e os nutricionistas. No seguimento foi apresentado um breve resumo sobre a aplicação deste tipo de pesquisa em estudos na área.

A aplicação desta metodologia apresenta-se como particularmente relevante para a investigação desenvolvida na área das ciências da nutrição e alimentação e para a expansão do conhecimento. Em Portugal, não existem ainda, estudos formais sobre a aplicação deste tipo de metodologia em trabalhos científicos desenvolvidos na área da alimentação e nutrição, mas a evidência empírica parece indicar que a pesquisa qualitativa enfrenta ainda muitas resistências, quer ao nível do conhecimento pelos nutricionistas, quer da sua aplicação nos estudos realizados a nível académico ou publicados na literatura.

Esta realidade apresenta-se de forma paradoxal face às características do fenómeno alimentar e nutricional e à confiabilidade deste tipo de pesquisa, quando desenvolvida corretamente, impondo-se a questão: "Porque é que a área das ciências da nutrição e alimentação, de cunho tão humanista e intersubjetivo em suas atividades, ainda tem fortes resistências para conhecer e reconhecer a cientificidade e aplicabilidade da metodologia qualitativa?"

As breves referências explanatórias presentes neste artigo, sobre a aplicabilidade da metodologia qualitativa apontam para um movimento mundial, muito atual, ao nível das pesquisas desenvolvidas na área da saúde em geral e da nutrição em específico, que buscam um conhecimento mais amplo dos fenómenos estudados. A pesquisa qualitativa, quando desenhada e desenvolvida rigorosamente, entre muitas outras áreas de aplicação, possibilita a compreensão aprofundada do comportamento alimentar, sendo útil ao desenho e avaliação de projetos e programas para a comunidade, e revela as experiências dos pacientes, familiares e profissionais de saúde envolvidos com o cuidado alimentar e nutricional, oferecendo uma visão mais assertiva, e humana do cuidar.

Perante a importância deste tipo de pesquisa e a realidade atual das ciências da nutrição e alimentação perante a mesma, parece-nos que o estabelecimento de mecanismos de educação/formação nesta temática e a aplicação de processos de colaboração interdisciplinar na realização de estudos científicos poderão ser estratégias importantes, para o desenvolvimento da utilização desta metodologia, na área das ciências da nutrição e alimentação, e a subsequente produção de conhecimento específico nesta área. 


\section{REFERÊNCIAS BIBLIOGRÁFICAS}

1. Denzin NK and Lincoln Y. Introduction - The discipline and practice of qualitative research. In Denzin NK and Lincoln Y(eds) The Sage Handbook of Qualitative Research. 3 ed. London: Sage Publications; 2005, pp. 1-32.

2. Swift JA, Tischler V. Getting started in qualitative research. J Hum Nutr Diet. 2010; 23: $559-566$

3. Harris JE, Gleason PM, Sheean PM et al. An introduction to qualitative research for food and nutrition professionals. J Am Diet Assoc. 2009; 109: 80-90.

4. Draper AK. The principles and application of qualitative research. Proc Nutr Soc. 2004; 63, 641-646.

5. Richardson JC, Liddle J. Where does good quality qualitative health care research get published?. Prim Health Care Res Dev. 2017; 18: 515-521.

6. Vieira CM, Turato ER. Percepções de pacientes sobre alimentação no seu processo de adoecimento crônico por síndrome metabólica: um estudo qualitativo. Rev Nutr. 2010; 23 (3): 425-432.

7. Koehnlein EA, Salado GA, Yamada AN. Adesão à reeducação alimentar para perda de peso: determinantes, resultados e a percepção do paciente. Rev Bras Nutr Clín. 2008; 23(1): 56-65

8. Farahmand M, Amiri P, Tehrani FR, et al. What are the main barriers to healthy eating among families? A qualitative exploration of perceptions and experiences of Tehranian men. Appetite. 2015; 89: 291-297.

9. Naithani S, Whelan K, Thomas J, et al. Hospital inpatients' experiences of access to food: a qualitative interview and observational study. Health Expect. 2008; 11: 294-303. 10. Denzin NK and Lincoln YS. The Sage handbook of qualitative research. 4 ed. London: Sage Publication; 2008.

11. Minayo MCS. O desafio do conhecimento: pesquisa qualitativa em saúde. 14 ed. São Paulo: Hucitec; 2014.

12. Bogdan R, Biklen S. Investigação qualitativa em educação: uma introdução à teoria e aos métodos. Porto: Porto Editora; 2010.

13. Anderson A. Una introducción a la investigación cualitativa. Rev Peru Psiq. 2000; 6(1):103-112

14. Turato ER. Tratado da metodologia da pesquisa clinica-qualitativa. 6 ed. Petrópolis: Vozes; 2013

15. Triviños ANS. Introdução à Pesquisa em Ciências Sociais: a pesquisa qualitativa em educação. 1 ed. São Paulo: Atlas; 2017.

16. Ludke M, André MEDA. Pesquisa em educação: abordagens qualitativas. 2ed. São Paulo: E.P.U.;2013.

17. Onocko-Campos R. Fale com eles! O trabalho interpretativo e a produção de consenso na pesquisa qualitativa em saúde: inovações a partir de desenhos participativos. Physis. 2011; 21(4): 1269-1286.

18. Correia T. Interpretação e validação científica em pesquisa qualitativa. Interface. 2013; 15(45): 263-274.

19. Lakatos EM, Marconi MA. Fundamentos de metodologia científica. 7 ed. São Paulo: Atlas; 2010.

20. Minayo MCS. Pesquisa social: teoria, método e criatividade. 32 ed. São Paulo: Vozes; 2012.

21. Malinowski B. Uma teoria científica da cultura. 1ed. Rio de Janeiro: Zahar; 1975 22. Mack N, Woodsong KMQ, Guest G et al. Participant Observation. In: Family Health International (eds) Qualitative Research Methods: a data collector's field guide. $1^{\text {a }} \mathrm{ed}$. North E3: Family Health International Publications;2005.p: 13-29.

23. Mack N, Woodsong KMQ, Guest G et al. Focus Group. In: Family Health International (eds) Qualitative Research Methods: a data collector's field guide. $1^{\mathrm{a}} \mathrm{ed}$. North Carolina: Family Health International Publications; 2005,p: 51-81.

24. Denzin NK, Lincoln YS. Paradigms and perspectives in transition. In: Denzin NK, Lincoln YS (eds). Handbook of Qualitative Research. 2nd ed. Thousand Oaks, California Sage; 2000. p:157-162.

25. Miles MB and Huberman AM. Data management and analysis methods. In: Denzin NK and Lincoln YS (eds) Handbook of Qualitative Research. 2nd ed. Thousand Oaks, California: Sage; 1994, pp. 428-444.

26. Coutinho C. Avaliação da qualidade da investigação qualitativa: algumas considerações teóricas e recomendações práticas. In: Souza FN, Souza DN, Costa AP (eds). Investigação Qualitativa - Inovação, Dilemas e Desafios. $1^{\text {a }}$ ed. Aracaju, Sergipe: Editora Tiradentes; 2015.p: 103-124.

27. EQUATOR Network - Enhancing the Quality and Transparency Of health Research [webpage]. Oxford: EQUATOR Network; [atualizado em 2017 Nov 3; citado em 2018 Fev 22]. Disponível em: http://www.equator-network.org

28. Tong A, Sainsbury P, Craig J. Consolidated criteria for reporting qualitative research (COREQ): a 32-item checklist for interviews and focus groups. Int J Qual Health Care. 2007; 19(6): 349-357.

29. O'Brien BC, Harris IB, Beckman TJ, et al. Standards for reporting qualitative research: a synthesis of recommendations. Acad Med. 2014; 89(9):1245-1251.

30. Canesqui AM. Pesquisas qualitativas em nutrição e alimentação. Rev Nutr. 2009; 22(1): 125-139.

31. Boog MCF. A Pesquisa qualitativa no campo da alimentação e nutrição. In: Barros AJP, Cecatti JG, Turato ER (eds). Pesquisa qualitativa em saúde: múltiplos olhares. Campinas:Komedi; 2005. p: 97-108.

32. Zoellner J, Harris JE. Mixed-Methods Research in Nutrition and Dietetics. J Acad Nutr Diet. 2017; 117(5): 683-697. 